\title{
Comparison of Clinical Characteristics and Outcome of Critically Ill Patients Admitted to Tertiary Care Intensive Care Units in India during the Peak Months of First and Second Waves of COVID-19 Pandemic: A Retrospective Analysis
}

\author{
Sukhyanti Kerai ${ }^{1} \odot$, Rahil Singh ${ }^{2} \odot$, Shanta Dutta $^{3} \odot{ }^{\circ}$ Aayushi Mahajan $^{4} \odot$, Munisha Agarwal $^{5}$
}

\section{Abstract}

Background: Coronavirus disease-2019 (COVID-19) continues to pose serious challenges to healthcare systems globally with the disease progressing over time in crest-trough pattern of waves. We compared the patient characteristics and outcomes of critically ill patients admitted during the first and second waves of COVID-19 pandemic.

Materials and Methods: We did a retrospective analysis of medical records of critically ill patients admitted to intensive care unit (ICU) at the peak period of both waves. The data on demographics, symptoms, treatment received, and outcomes of patients were recorded.

Results: Compared to first wave, significantly more females, younger age group, and those without underlying comorbidities required ICU admission during the second wave. The treatments received during both periods were similar except for preferential use of methylprednisolone over dexamethasone and proclivity of bilevel positive airway pressure (BiPAP) ventilation over high-flow nasal cannula (HFNC). There was no significant difference in the duration of ICU stay and mortality of patients. During the first wave, the factors associated with nonsurvival of patients were advanced age, comorbidities, severe disease, and a lesser number of days on HFNC. All these factors along with higher Sequential Organ Failure Assessment (SOFA) score were observed to be linked with patient nonsurvival during the second wave.

Conclusion: In India, the second wave of COVID-19 significantly influenced ICU demographics with a predominance of females and young adults requiring critical care. During both time periods, patients received similar treatment except for the propensity to use methylprednisolone and BiPAP as opposed to dexamethasone and HFNC in second wave. No significant difference in ICU mortality was noted.

Keywords: Coronavirus disease-2019, Coronavirus disease-2019 intensive care unit, Coronavirus disease-2019 in India, Mortality, Predictors, Severity.

Indian Journal of Critical Care Medicine (2021): 10.5005/jp-journals-10071-24046
\end{abstract}

\section{INTRODUCTION}

The second wave of coronavirus disease-2019 (COVID-19) pandemic in India, in recent weeks, has overwhelmed the healthcare system, leaving hospitals struggling to cope with limited availability of critical care beds, vital drugs, and oxygen. ${ }^{1}$ Anecdotal report suggests that this second wave differs from the first with respect to factors such as age range and severity of the disease. ${ }^{2}$ During this wave, two important dominant variants have been identified: novel B.1.617 (Indian variant or delta variant) and B.1.1.7 variant (United Kingdom (UK) variant). These variants have been labeled as "variant of concern" by the World Health Organization. These variants have been found to be associated with increased infectivity. There is, however, uncertainty regarding an increased severity of these newer strains compared to previous ones. $^{3,4}$

Compared to the first wave of COVID-19 last year, when the second wave was encountered by many countries globally, there was a better understanding of COVID-19 pathophysiology with significant treatment modification including systematic and early administration of glucocorticoids as well as intermediate-/full-dose thromboprophylaxis. However, despite these advantages, data from European countries showed no improvement in ICU mortality between first and second waves of pandemic. ${ }^{5,6}$
1,2Department of Anaesthesiology and Critical Care, Maulana Azad
Medical College, New Delhi, India
${ }^{3-5}$ Department of Anaesthesiology and Intensive Care, Maulana Azad
Medical College, New Delhi, India
Corresponding Author: Rahil Singh, Department of Anaesthesiology
and Critical Care, Maulana Azad Medical College, New Delhi, India,
Phone: +91 9810719025, e-mail: drrahilsingh@gmail.com
How to cite this article: Kerai S, Singh R, Dutta S, Mahajan A, Agarwal M.
Comparison of Clinical Characteristics and Outcome of Critically III
Patients Admitted to Tertiary Care Intensive Care Units in India during
the Peak Months of First and Second Waves of COVID-19 Pandemic: A
Retrospective Analysis. Indian J Crit Care Med 2021;25(12):1349-1356.

Source of support: Nil

Conflict of interest: None

There is a paucity of data from India regarding the comparison of disease severity and characteristics of COVID-19 between first and second waves of COVID-19 pandemic. We therefore conducted a retrospective analysis of critically ill COVID-19 patients admitted to intensive care units (ICUs) at our hospital at the time of peaks of first and second waves of pandemic.

(O) The Author(s). 2021 Open Access This article is distributed under the terms of the Creative Commons Attribution 4.0 International License (https://creativecommons. org/licenses/by-nc/4.0/), which permits unrestricted use, distribution, and non-commercial reproduction in any medium, provided you give appropriate credit to the original author(s) and the source, provide a link to the Creative Commons license, and indicate if changes were made. The Creative Commons Public Domain Dedication waiver (http://creativecommons.org/publicdomain/zero/1.0/) applies to the data made available in this article, unless otherwise stated. 


\section{Materials and Methods}

This retrospective study comprised of patients admitted to our 27 bed ICUs for acute respiratory failure due to COVID-19 during the first wave (from April 1 to April 30, 2021) and second wave (from August 15 to September 15,2020). These time periods corresponded to highest peak of the second and first waves of COVID-19 pandemic in India. Institutional ethical committee approval was obtained prior to commencing data collection from medical records of patients. The inclusion criteria for this study were laboratory-confirmed COVID-19 patients requiring ICU admission. Positive reverse transcriptase-polymerase chain reaction (RT-PCR) test from nasal or pharyngeal swab samples was utilized to confirm diagnosis of COVID-19 infection. The indication for ICU admission was respiratory failure due to COVID-19 infection that required respiratory support with high-flow nasal cannula (HFNC), bilevel positive airway pressure (BiPAP) ventilation, or mechanical ventilation to maintain oxygen saturation of more than $94 \%$ with no clinical evidence of enhanced work of breathing (tachypnea, use of accessory muscles of respiration). Patients were admitted to ICUs either directly from emergency department or shifted from inpatient hospital wards. We excluded those with suspected COVID-19 infection but had no laboratory confirmation and patients who expired within 24 hours of admission to ICU as many of these cases expired before any treatment was instituted.

\section{Treatment Protocol in ICUs}

All patients requiring oxygen support over $15 \mathrm{~L} /$ minute with reservoir mask and with respiratory rate above 30/minute were admitted to ICUs. Once in ICU, these patients were provided HFNC or BiPAP ventilation at the discretion of attending intensivists. Mechanical ventilation was instituted on failure of HFNC or BiPAP to maintain adequate oxygenation or ventilation.

The drug treatment protocol framed by a multidisciplinary expert committee in the hospital was followed in ICUs. It was based on the Indian Council of Medical Research (ICMR) recommendations with modifications and timely update on the basis of evidence generated through clinical trials and international consensus guidelines.

Unless contraindicated, remdesivir was the preferred antiviral agent. If any contraindications for its administration were present, ivermectin was being given at discretion of treating physician. All patients admitted to ICU were given parenteral corticosteroid. The steroid used was either dexamethasone or methylprednisolone chosen at a preference of treating doctor. Tocilizumab was used for patients with increasing oxygen requirement despite being on steroids and a tenfold increase in interleukin- 6 levels (normal limit: $0-7 \mathrm{pg} / \mathrm{mL}$ at our center) and increase in other inflammatory markers. This cutoff value of IL- 6 was decided based on a study by Herold et al. published at the beginning of pandemic. ${ }^{7}$ Anticoagulation therapy involved low-dose molecular weight heparin (LMWH) and in cases of renal derangement subcutaneous unfractionated heparin (UFH)was administered. During the first wave, convalescent plasma was given to patients with severe COVID-19 infection presenting within 7 days of symptom onset; however, during the second wave, it was not favored for the treatment of ICU patients; as subsequent literature concluded that critically ill patients are unlikely to gain from plasma transfusion and to achieve best diagnosis, it should be preferably within 3 days after diagnosis. ${ }^{8}$ The practice of administration prophylactic anticoagulation was also differed during both waves of pandemic. During the first wave, prophylactic dose of LMWH or UFH was given to severe cases admitted to ICU, whereas weight-based intermediate dose of anticoagulation was administered during the second wave, provided that there are no risk factors for bleeding., ${ }^{9,10}$

Other drugs included were prophylactic/therapeutic antibiotics as per local policy, stress ulcer prophylaxis, nutritional support, vitamins/antioxidant supplements, and drugs according to the underlying comorbidities.

\section{Data Collection}

The variables collected from medical records are demographic characteristics, comorbidities, symptoms on presentation to hospital, duration from onset of symptoms to hospitalization, baseline oxygen saturation $\left(\mathrm{SpO}_{2}\right)$ as measured by noninvasive pulse oximeter, ratio of partial pressure of oxygen to fraction of inspired air on arterial blood gas analysis $\left(\mathrm{PaO}_{2} / \mathrm{FiO}_{2}\right)$, sequential organ failure assessment (SOFA) score, report of blood investigations on day 1 of ICU, respiratory support provided along with a number of days spent on different modalities, COVID-19 treatment provided in ICU, duration of ICU stay, and patient outcomes.

\section{Statistical Analysis}

The qualitative variables (such as demographic characteristics) were presented as frequencies and percentages (\%). The quantitative variables (normally distributed continuous variables) were expressed as mean and standard deviation. The data were analyzed by using SPSS standard software version 25 . The comparison of normally distributed continuous variables between the groups was performed using Student's $t$ test. Nominal categorical data between the groups were compared using Pearson's Chi-squared test. Non-normal distribution continuous variables were compared using Mann-Whitney $U$ test. For all statistical tests, a $p$ value less than 0.05 will be taken to indicate a significant difference.

\section{RESULTS}

During the peak period of the first wave of COVID-19, a total of 147 patients were admitted to ICUs; after screening their records 104 patient were taken for final analysis. A total number of patients admitted to ICUs during the peak month of second wave were 163 , out of which 116 met our inclusion criteria.

Compared to the first wave, the patients requiring ICU admissions were younger and those with no comorbidities (Figs 1 and 2). The mean age of patients was $56.8 \pm 16.3$ and $49.5 \pm 13.5$ years, respectively, during the first and second waves of COVID-19 ( $p=0.00)$. Earlier ICU admission of female patients was around $38 \%$, which increased to $55.5 \%$ in the second wave. Underlying comorbid conditions were observed in $72.6 \%$ of ICU admissions in the first wave as opposed to $54 \%$ in the second one. There are a significantly greater number of patients with coronary artery disease, hypertension, diabetes, renal dysfunction during the first wave as opposed to the second. No difference in the duration of symptoms before hospitalization was found between the two groups. Fever ( 90.4 vs $77.8 \% ; p=0.01)$, malaise (33.6 vs $22.1 \% ; p=0.05)$, cough (70.6 vs $46.1 \% ; p=0.000$ ), and headache (82.4 vs $22.4 \% ; p=0.00$ ) were noted to as presenting complaint/s in a significantly greater proportion of patients in the second wave (Fig. 3). The baseline saturation of oxygen was significantly lower in ICU patients during the second wave $(73.6 \pm 16.9$ vs $79.29 \pm 12.7 ; p=0.006)$. No difference in baseline SOFA score was found between the two cohorts. Regarding respiratory support 


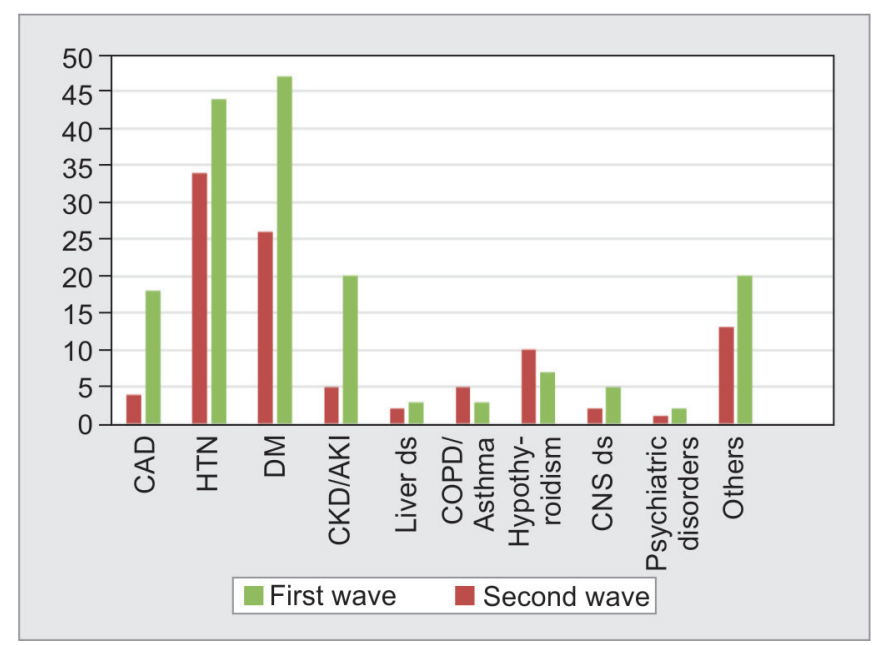

Fig. 1: Comparison of comorbidities of ICU patients during first and second waves of COVID-19

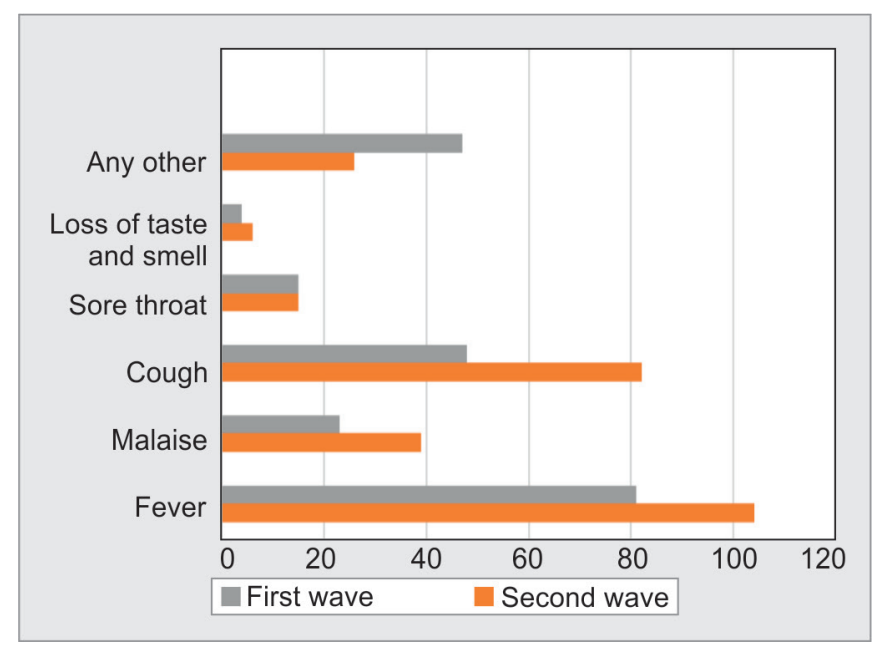

Fig. 2: Comparison of symptoms on presentation of patients during first and second wave of COVID-19

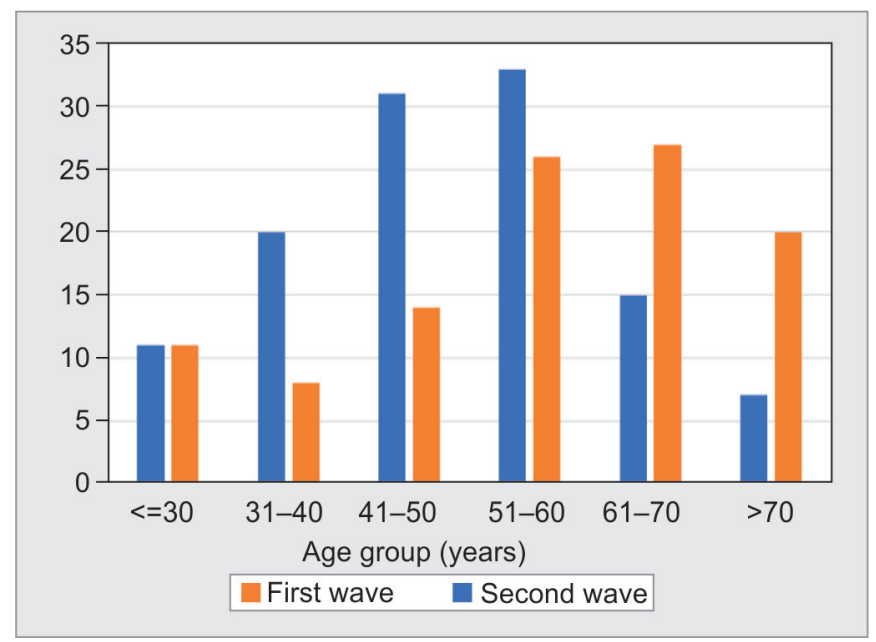

Fig. 3: Comparison of age groups of patients admitted in ICU during first and second wave of COVID-19 utilization between the two groups, a significantly lesser number of days during ICU stay were spent on HFNC during the second wave and patients remained on BiPAP for a greater number of days. In contrast, during the first wave, a greater number of ICU days were on HFNC. There was no difference in the number of days spent on mechanical ventilation (MV) during ICU stay of patients. No dissimilarity in the utilization of remdesivir was seen during the first and second waves. However, methylprednisolone was the frequent steroid utilized in the second wave as compared to dexamethasone during the first wave although an insignificantly but greater number of patients admitted during the first wave received tocilizumab injection. Compared to the peak month of second wave, a greater number of ICU patients required dialysis during the first wave. There was no difference in baseline laboratory parameters except for values of blood urea, serum creatinine, and prothrombin time which were observed to be higher in patients admitted in the first wave. The duration of ICU stay and mortality of patients were comparable in both the groups (Table 1).

During the peak time the of second wave of COVID-19, advanced age; administration of vasopressors; lesser number of days spent on HFNC and MV; lesser number of days of remdesivir; higher SOFA score; lower $\mathrm{PaO}_{2} / \mathrm{FiO}_{2}$ ratio; and other hematological derangements including higher total leukocyte counts, higher alkaline phosphatase, and higher serum creatinine values on day 1 of ICU admission were observed to be present in nonsurvivors compared to survivors (Table 2).

At the peak time period of first wave, nonsurvivors compared to survivors had older age, comorbidities, received vasopressors, spent lesser number of days on HFNC and MV along with lower $\mathrm{PaO}_{2} / \mathrm{FiO}_{2}$, and platelet count (Table 3).

\section{Discussion}

Since the middle of March 2021, India experienced a devastating second wave of COVID-19 pandemic, and with exponential surge in daily cases, it reached the summit by late April 2021. During the second wave, the daily positivity rate increased from $1.62 \%$ on March 1, 2021, to $20 \%$ on May 13, 2021. Noncompliance with COVID-19 appropriate behavior, failure to curb mass gatherings, and emergence of a new variant of severe acute respiratory syndrome coronavirus 2 (SARS-CoV-2) was largely attributed as critical factors for mammoth upsurge of the second wave of COVID-19. ${ }^{11}$ In contrast, after identification of index case on January 27, 2020, the first wave of COVID-19 in India slowly rose over 6 months and peaked in mid-September 2020 before receding slowly.

Compared to the first wave, certain demographics of the critically ill patients during the second wave were observed to be different. We found significantly more females, younger age group, and those without any comorbidities required ICU admission during the second wave peak. Emergence of a new strain of mutated virus having high transmissibility and poor adherence to COVID-19 protocol along with lack of immunization in the younger population might have resulted in contagion in young healthy adults. The variant B.1.617.2 of SARS-CoV-2 was reported as the primary strain responsible for COVID-19 cases in India during the second wave. Data indicate that it is $40-60 \%$ more transmissible than the original strain of virus and resulted in an increased risk of hospitalization in affected individual. ${ }^{12}$ Despite reports of females being more compliant with COVID-19 appropriate behavior and restraining 
Table 1: Comparison of demographics, ICU treatment received, outcomes, and laboratory parameters of patients admitted during the first and second waves of COVID-19 pandemic

\begin{tabular}{|c|c|c|c|}
\hline & First wave & Second wave & pvalue \\
\hline Gender (\%) & & & 0.008 \\
\hline Male & 62.2 & 44.4 & \\
\hline Female & 37.7 & 55.5 & \\
\hline Comorbidities (\%) & 72.6 & 52.9 & 0.002 \\
\hline Age (in years; mean \pm S.D.) & $56.8 \pm 16.3$ & $49.5 \pm 13.5$ & 0.00 \\
\hline Duration of illness before hospitalization (days) & $5.3 \pm 2.9$ & $5.3 \pm 2.3$ & 0.025 \\
\hline $\mathrm{SpO}_{2}(\%)$ & $79.29 \pm 12.7$ & $73.6 \pm 16.9$ & 0.006 \\
\hline SOFA score & $3.5 \pm 2.2$ & $3.4 \pm 1.3$ & 0.72 \\
\hline HFNC (number of days; mean \pm S.D.) & $2.88 \pm 3.6$ & $0.85 \pm 2.1$ & $<0.001$ \\
\hline BiPAP (number of days; mean \pm S.D.) & $2.6 \pm 3.2$ & $4.4 \pm 4.8$ & 0.001 \\
\hline MV (number of days; mean \pm S.D.) & $1.5 \pm 3.1$ & $1.9 \pm 2.7$ & 0.274 \\
\hline Remdesivir (number of days; mean \pm S.D.) & $3.5 \pm 2.8$ & $4.1 \pm 2.1$ & 0.04 \\
\hline Dexamethasone (number of days; mean \pm S.D.) & $6.14 \pm 4.4$ & $4.1 \pm 2.1$ & $<0.001$ \\
\hline MPS (number of days; mean \pm S.D.) & $2.19 \pm 3.9$ & $5.9 \pm 6.1$ & $<0.001$ \\
\hline Tocilizumab (\% of patients) & 11.3 & 4.2 & 0.89 \\
\hline Administration of vasopressors (\% of patients) & 11.3 & 7.7 & 0.34 \\
\hline Administration of dialysis (\% of patients) & 11.3 & 1.7 & 0.00 \\
\hline Duration of ICU stay (number of days; mean \pm S.D.) & $7.2 \pm 5.7$ & $6.8 \pm 6.3$ & 0.65 \\
\hline Nonsurvivors (\% of patients & 53.2 & 56.3 & 0.43 \\
\hline \multicolumn{4}{|l|}{ Investigations } \\
\hline Hemoglobin (g/dL) & $11.8 \pm 2.3$ & $12.2 \pm 2.10$ & 0.206 \\
\hline Total leukocyte count $\left(\times 10^{9} / \mathrm{L}\right)$ & $14517.1 \pm 2369$ & $10576.2 \pm 3931$ & 0.07 \\
\hline Neutrophils (\%) & 89.1 & 83.6 & \\
\hline Lymphocytes (\%) & 8 & 12 & \\
\hline Platelet count (lakh per unit volume) & $1.89 \pm 0.9$ & $1.93 \pm 0.9$ & 0.766 \\
\hline Total bilirubin (mg/dL) & $0.7 \pm 0.5$ & $0.8 \pm 0.3$ & 0.54 \\
\hline Alanine aminotransferase (U/L) & $87.8 \pm 197.4$ & $127.3 \pm 581.4$ & 0.50 \\
\hline Alanine aminotransferase (U/L) & $83.4 \pm 95.9$ & $151.7 \pm 660.2$ & 0.29 \\
\hline Alanine phosphatase (U/L) & $133.9 \pm 81.5$ & $133.29 \pm 96.1$ & 0.88 \\
\hline Blood urea (mg/dL) & $67.4 \pm 60.4$ & $43.1 \pm 22.6$ & 0.000 \\
\hline Serum creatinine $(\mathrm{mg} / \mathrm{dL})$ & $1.60 \pm 2.74$ & $0.72 \pm 0.39$ & 0.001 \\
\hline Sodium (mEq/L) & $140.1 \pm 19.1$ & $136.9 \pm 5.1$ & 0.08 \\
\hline Potassium (mEq/L) & $4.6 \pm 0.8$ & $4.6 \pm 0.5$ & 0.77 \\
\hline INR & $1.16 \pm 0.35$ & $1.02 \pm 0.16$ & 0.00 \\
\hline D-dimer (ng/mL) & $1817.1 \pm 1542.5$ & $1530.7 \pm 1472.1$ & 0.165 \\
\hline $\mathrm{PaO}_{2} / \mathrm{FiO}_{2}$ & $88.4 \pm 49.7$ & $85.2 \pm 35.5$ & 0.57 \\
\hline
\end{tabular}

public policy, significantly more females required admission to ICU indicating higher infectivity of new virus strain. ${ }^{13}$

Consistent with experiences of other countries during the first wave, older patients with underlying comorbidities more likely to be admitted to the ICU with a high mortality rate. ${ }^{14}$ Among the comorbid conditions in patients admitted to ICUs during the first wave, hypertension was found to be more significantly prevalent among nonsurvivors. Previous studies have demonstrated that after adjusting for age and sex, hypertension showed a significant correlation with increased disease severity and mortality. ${ }^{15,16}$ The exact mechanism for association of hypertension with severity of COVID-19 infection is not clear. One of the plausible explanations is interaction of both hypertension and SARS-CoV-2 with angiotensin converting enzyme 2 (ACE2) receptors. There is a high affinity of SARS-CoV-2 for binding to ACE2 receptors to gain entry to host cells. ACE2 is a modulator of renin-angiotensin-aldosterone system, which regulates blood pressure and fluid balance and has a role in pathogenesis of hypertension.

There is nuance change in symptoms on presentation to hospital between patients of first and second waves of COVID-19. During second wave of COVID-19 pandemic significantly greater number of ICU patients presented with headache, fever, malaise and cough.Similar finding has been reported by data from a self-reporting mobile application from the UK. ${ }^{17}$ Evolution of virus strain, predominance of younger patients, and initiation of vaccination drive could be adduced to change in symptomatology of infection. Loss of smell or anosmia has been identified as one of the frequent symptoms of COVID-19 and has been shown to be strongly linked to positive SARS-CoV-2 test. ${ }^{18}$ The prevalence of anosmia in COVID-19, however, varies greatly between population depending on host factors and differences in mutations of spike protein of virus. In the first 6 months of pandemic, two strains of 
Table 2: Comparison of demographics, ICU treatment received, outcomes, and laboratory parameters of survivor and nonsurvivor patients admitted during the second wave of COVID-19 pandemic

\begin{tabular}{|c|c|c|c|}
\hline & Nonsurvivors & Survivors & $p$ value \\
\hline Gender (\%) & & & 0.09 \\
\hline Male & 45.4 & 42.5 & \\
\hline Female & 54.5 & 57.5 & \\
\hline Comorbidities (\%) & 54.5 & 50 & 0.64 \\
\hline Administration of vasopressor during ICU stay (\%) & 11.6 & 0 & 0.02 \\
\hline Use of dialysis (\%) & 1.2 & 2.5 & 0.79 \\
\hline Age (in years; mean \pm S.D.) & $51.3 \pm 14.4$ & $45.9 \pm 10.9$ & 0.04 \\
\hline Duration of symptoms before hospitalization (in days; mean \pm S.D.) & $5.4 \pm 2.4$ & $5.3 \pm 2.3$ & 0.85 \\
\hline $\mathrm{SpO}_{2}$ on presentation to ICU (in \%; mean \pm S.D.) & $72.8 \pm 15.6$ & $75.1 \pm 19.3$ & 0.48 \\
\hline Number of days patients received BiPAP during ICU stay (in days; mean \pm S.D.) & $4.4 \pm 4.3$ & $4.2 \pm 5.8$ & 0.81 \\
\hline Number of days patients received HFNC during ICU stay (in days; mean \pm S.D.) & $0.4 \pm 0.9$ & $1.7 \pm 3.2$ & 0.001 \\
\hline Number of days patients received MV during ICU stay (in days; mean \pm S.D.) & $2.9 \pm 2.9$ & $0.05 \pm 0.2$ & $<0.001$ \\
\hline Number of days patients received Remdesivir during ICU stay (in days; mean \pm S.D.) & $3.7 \pm 2.4$ & $4.8 \pm 0.9$ & 0.009 \\
\hline Number of days patients received dexamethasone during ICU stay (in days; mean \pm S.D.) & $2.9 \pm 4.1$ & $3.5 \pm 4.6$ & 0.45 \\
\hline Number of days patients received methylprednisolone during ICU stay (in days; mean \pm S.D.) & $5 \pm 4.8$ & $7.6 \pm 7.8$ & 0.03 \\
\hline Number of patients received receiving tocilizumab (\%) & 3.8 & 5 & 0.76 \\
\hline SOFA score at ICU admission & $3.6 \pm 1.3$ & $2.9 \pm 1.1$ & 0.008 \\
\hline Hemoglobin (g/dL) & $12.1 \pm 2.1$ & $12.2 \pm 2.0$ & 0.80 \\
\hline Total leukocyte count $\left(\times 10^{9} / \mathrm{L}\right)$ & $11121 \pm 4023$ & $9500 \pm 3550$ & 0.03 \\
\hline Polymorphs (\%) & $84.7 \pm 8.8$ & $81.5 \pm 6.9$ & 0.04 \\
\hline Lymphocytes (\%) & $11.4 \pm 7.3$ & $13.4 \pm 5.3$ & 0.13 \\
\hline Monocytes (\%) & $2.9 \pm 2.7$ & $3.0 \pm 1.9$ & 0.89 \\
\hline Eosinophils (\%) & $0.9 \pm 1.5$ & $0.8 \pm 0.8$ & 0.77 \\
\hline Platelet count (lakh per unit volume) & $1.84 \pm 0.8$ & $2.1 \pm 1.1$ & 0.14 \\
\hline Total bilirubin (mg/dL) & $0.8 \pm 0.3$ & $0.7 \pm 0.2$ & 0.19 \\
\hline Alanine aminotransferase (U/L) & $163.9 \pm 711.9$ & $55.1 \pm 36.2$ & 0.34 \\
\hline Aspartate aminotransferase (U/L) & $198.7 \pm 807.3$ & $58.8 \pm 34.5$ & 0.28 \\
\hline Alanine phosphatase (U/L) & $147.4 \pm 111.6$ & $102.4 \pm 40.7$ & 0.01 \\
\hline Blood urea (mg/dL) & $44.7 \pm 25.5$ & $40.1 \pm 15.1$ & 0.30 \\
\hline Serum creatinine (mg/dL) & $0.8 \pm 0.4$ & $0.5 \pm 0.1$ & 0.001 \\
\hline Sodium (mEq/L) & $137 \pm 5.6$ & $136.7 \pm 3.9$ & 0.76 \\
\hline Potassium (mEq/L) & $4.7 \pm 0.6$ & $4.5 \pm$ & 0.06 \\
\hline INR & $1.02 \pm 0.18$ & $1.03 \pm 0.12$ & 0.88 \\
\hline D-dimer (ng/mL) & $1562.1 \pm 1450.4$ & $1468.6 \pm 1531.4$ & 0.74 \\
\hline $\mathrm{PaO}_{2} / \mathrm{FiO}_{2}$ & $78.3 \pm 32.9$ & $98.7 \pm 37.05$ & 0.003 \\
\hline
\end{tabular}

SARS-CoV-2 resulting from mutation in spike protein D614 and G614 coexisted in most of the population. Infection with D614 rarely led to more than $10 \%$ anosmia prevalence, whereas $\mathrm{G} 614$ prevalence led to prevalence of $10-90 \%$ in the same ethnicity of patients. ${ }^{19}$ Although few anecdotal reports suggest that the prevalence of anosmia is lesser during the second wave of pandemic in India, we noted that during both waves of pandemic, a similar proportion of patients presented to ICU with anosmia. The difference in the mutation of virus strain could be plausible explanation for disparity between reports and the present study.

As the peak of first wave of COVID-19 in India progressed more slowly compared to Western countries by the time it achieved peak, several evidence-based therapeutic strategies were identified such as steroid for hypoxemic patients, role of thromboprophylaxis and antiviral drug remdesivir. Our study cohorts received all these groups of drugs during both waves. One of the differences observed was the change in the preference for type of steroid medication during waves. Following publication of RECOVERY trial, dexamethasone became standard of care. ${ }^{20}$ Our institutional policy does not advocate any specific steroid for COVID-19 patients and type and dose of steroids are decided on discretion of attending intensivists. Despite availability of both dexamethasone and methylprednisolone, we found that patients during the second wave received methylprednisolone for longer duration in comparison with dexamethasone indicating its preference by intensivists. Recent studies have now gathered evidence that in COVID-19 patients requiring MV, sufficiently dosed methylprednisolone can lead to a further reduction in mortality as compared to dexamethasone. ${ }^{21,22}$

At the outset of COVID-19 pandemic, patients managed with invasive $\mathrm{MV}$ experienced mortality rate of $90 \% .{ }^{23} \mathrm{~A}$ recent meta-analysis on outcomes for COVID-19 requiring MV reported best possible case fatality rate (CFR) of $43 \%$ and a worst possible CFR of $64 \% .{ }^{24}$ Unprecedented surge in the number of cases along with 
Table 3: Comparison of demographics, ICU treatment received, outcomes, and laboratory parameters of survivor and nonsurvivor patients admitted during the first wave of COVID-19 pandemic

\begin{tabular}{|c|c|c|c|}
\hline & Expired & Survived & $p$ value \\
\hline Gender (\%) & & & 0.25 \\
\hline Male & 58.2 & 69.2 & \\
\hline Female & 41.7 & 30.7 & \\
\hline Comorbidities (\%) & 79 & 61.5 & 0.05 \\
\hline Administration of vasopressor during ICU stay (\%) & 16.4 & 2.6 & 0.03 \\
\hline Dialysis received (\%) & 11.9 & 10.2 & 0.87 \\
\hline Age (in years; mean \pm S.D.) & $60 \pm 16.2$ & $51.4 \pm 15.3$ & 0.008 \\
\hline Duration of symptoms before hospitalization (in days; mean \pm S.D.) & $5.2 \pm 3$ & $5.6 \pm 2.8$ & 0.85 \\
\hline $\mathrm{SpO}_{2}$ on presentation to ICU (in \%; mean \pm S.D.) & $78.2 \pm 13.7$ & $81 \pm 10.6$ & 0.28 \\
\hline Number of days patients received BiPAP during ICU stay (in days; mean \pm S.D.) & $2.8 \pm 3.1$ & $2.2 \pm 3.3$ & 0.40 \\
\hline Number of days patients received HFNC during ICU stay (in days; mean \pm S.D.) & $2.4 \pm 3.3$ & $3.6 \pm 4.1$ & 0.09 \\
\hline Number of days patients received MV during ICU stay (in days; mean \pm S.D.) & $2.4 \pm 3.6$ & $0.03 \pm 0.1$ & 0.00 \\
\hline Number of days patients received dexamethasone during ICU stay (in days; mean \pm S.D.) & $5.8 \pm 4.6$ & $6.6 \pm 4.1$ & 0.36 \\
\hline Number of days patients received methylprednisolone during ICU stay (in days; mean \pm S.D.) & $2.3 \pm 4.2$ & $1.8 \pm 3.4$ & 0.56 \\
\hline Number of days patients received remdesivir during ICU stay (in years; mean \pm S.D.) & $3.3 \pm 2.8$ & $3.7 \pm 2.6$ & 0.56 \\
\hline Number of patients receiving tocilizumab (\%) & 10.4 & 12.8 & 0.87 \\
\hline SOFA score at ICU admission (in mean \pm S.D.) & $3.7 \pm 2.6$ & $3.1 \pm 1.4$ & 0.19 \\
\hline Hemoglobin (g/dL) & $11.6 \pm 2.5$ & $12.0 \pm 2.2$ & 0.40 \\
\hline Total leukocyte count $\left(\times 10^{9} / \mathrm{L}\right)$ & $15944 \pm 29351.3$ & $12064.8 \pm 6717.2$ & 0.41 \\
\hline Polymorphs (\%) & $89.9 \pm 7.1$ & $87.9 \pm 6.7$ & 0.16 \\
\hline Lymphocytes (\%) & $7.9 \pm 6.5$ & $8.6 \pm 3.9$ & 0.16 \\
\hline Monocytes (\%) & $1.4 \pm 1.2$ & $2.0 \pm 1.9$ & 0.04 \\
\hline Eosinophils (\%) & $0.5 \pm 0.7$ & $0.9 \pm 1.7$ & 0.07 \\
\hline Platelet count (lakh per unit volume) & $2.1 \pm 1.0$ & $1.5 \pm 0.6$ & 0.002 \\
\hline Total bilirubin (mg/dL) & $0.7 \pm 0.6$ & $0.7 \pm 0.4$ & 0.66 \\
\hline Alanine aminotransferase (U/L) & $101.4 \pm 244.5$ & $64.6 \pm 54.4$ & 0.35 \\
\hline Aspartate aminotransferase (U/L) & $90.6 \pm 114.7$ & $71 \pm 48$ & 0.31 \\
\hline Alkaline phosphatase (U/L) & $138 \pm 93.2$ & $127 \pm 56.5$ & 0.50 \\
\hline Blood urea (mg/dL) & $73.7 \pm 70.7$ & $56.5 \pm 34.8$ & 0.15 \\
\hline Serum creatinine $(\mathrm{mg} / \mathrm{dL})$ & $1.7 \pm 3.1$ & $1.4 \pm 2.0$ & 0.62 \\
\hline Sodium (mEq/L) & $141.3 \pm 23.8$ & $138.1 \pm 4.1$ & 0.41 \\
\hline Potassium (mEq/L) & $4.6 \pm 0.8$ & $4.6 \pm 0.8$ & 0.68 \\
\hline INR & $1.19 \pm 0.3$ & $1.10 \pm 0.3$ & 0.22 \\
\hline D-dimer $(\mathrm{ng} / \mathrm{mL})$ & $2074.9 \pm 1672.8$ & $1378.1 \pm 1187.4$ & 0.19 \\
\hline $\mathrm{PaO}_{2} / \mathrm{FiO}_{2}$ & $83.6 \pm 54.1$ & $96.7 \pm 40.4$ & 0.02 \\
\hline
\end{tabular}

strained critical care facilities in both high-income and low-middleincome countries made avoidance of intubation a critical issue. ${ }^{25}$ Data from UK suggested that over the time since the beginning of pandemic, there was a trend of reduced use of invasive ventilator over noninvasive modalities despite lower median $\mathrm{PaO}_{2} / \mathrm{FiO}_{2}$ ratio in ICU patients. ${ }^{26}$ In our center, noninvasive respiratory support was preferred initially in critically ill COVID-19 cases and MV was used towards later period of ICU stay. We found that during both waves of COVID-19, ICU patients remained on noninvasive respiratory support modalities for longer time periods compared to invasive MV. The use of HFNC was curtailed during the peak of the second wave due to severe shortage of oxygen. There was higher proportion of survival in ICU patients in whom BiPAP or HFNC were used as respiratory support. At the beginning of pandemic, noninvasive modalities were avoided due to potential risk of aerosolization of virus. However, an abundance of literature has surfaced, noting their relative safety of use. ${ }^{27,28}$ Various studies have showed NIV to be successful in majority of patients with moderate-to-severe COVID-19 acute respiratory distress syndrome (ARDS). ${ }^{29,30}$

The mortality of critically ill patients requiring ICU admission was statistically insignificant and was more than $50 \%$ during the peak months of the first and second waves. Although the difference was not significant, it was slightly higher during the second wave. This may be attributed to period of higher ICU load or demand, when the processes of care associated with ICU mortality such as staffing ratio, availability of experts and processes to reduce nosocomial infections were different. $^{31,32}$ The comparison of mortality of ICU patients during the first and second waves of COVID-19 from European countries also observed similar result. In Europe, most of the countries experienced the first wave of COVID-19 in the spring of 2020 and a second wave occurring in the later summer and autumn of 2020. During the second wave, several evidence-driven significant modifications in patient management were noted in these countries such as use of steroids, remdesivir, 
and tocilizumab, unrestrictive use of HFNC for hypoxemia. These changes were associated a decrease in 30-day mortality and ICU admissions in hospitalized patients. The ICU mortality, however, remains unchanged between first and second waves., ${ }^{5,6}$ These findings support importance of instituting optimal COVID-19 treatment before necessity of ICU treatment arises. This could be attributed to massive surge in number of cases during the second wave and lack of evidence for therapeutic effectiveness of any drug once severe lung damage ensued during the course of illness.

During the first wave, older age, comorbidities, use of mechanical ventilation, and lower $\mathrm{PaO}_{2} / \mathrm{FiO}_{2}$ ratio were found to be associated with higher patient mortality. These findings are consistent with previous studies. ${ }^{33,34}$ In contrast, no significant difference in underlying comorbid conditions during the second wave was observed between survivors and nonsurvivors. To mitigate the shortage of ICU beds during the second wave, the facilities for noninvasive modalities of respiratory support (mainly BiPAP) were created in locations outside ICUs in our center. This resulted in more sick patients with deranged laboratory parameters coming to ICUs. Compared to first wave, the nonsurvivor patients had higher SOFA score and derangements in blood investigations on the first day of ICU admission during the second wave.

The main limitations of the present study are its retrospective and single-center design. However, as we included data from single center, homogeneity in patient management during two periods was ensured. Another limitation is that the subgroup descriptive statistics were unadjusted for potential confounders. Despite these limitations, the size of cohorts included has allowed us to present a reasonably complete picture of critically ill COVID-19 patients as presented to our hospital.

\section{Conclusion}

Compared to the first wave of COVID-19, females, younger patients, and those without underlying comorbidities required ICU admission during the second wave. The majority of symptoms were similar in both periods, nevertheless higher incidence of headache, fever, cough, malaise, and cough were noted in the second wave. The patients during both periods received similar drug treatment and there was a trend of preferring noninvasive modalities of respiratory support. In both study periods, the duration of ICU stays and mortality rate remained the same.

\section{OrCID}

Sukhyanti Kerai 이 https://orcid.org/0000-0002-7771-0786

Rahil Singh ㄴ https://orcid.org/0000-0002-3941-1398

Shanta Dutta $\odot$ https://orcid.org/0000-0002-6371-9595

Aayushi Mahajan (1) https://orcid.org/0000-0002-5903-1157

Munisha Agarwal @ https://orcid.org/0000-0003-2958-6810

\section{References}

1. Kumar S. Second wave of COVID-19: emergency situation in India. J Travel Med 2021;28(7):taab082. Available from: DOI: 10.1093/jtm/ taab082.

2. Jain VK, lyenger KP, Vaishya R. Differences between first and second wave of COVID-19 in India. Diabetes Metab Syndr 2021;15(3):1047-1048. DOI: $10.1016 / j . d s x .2021 .05 .009$.
3. Grubaugh ND, Hodcroft EB, Fauver JR, Phelan AL, Cevik M Public health actions to control new SARS-CoV-2 variants. Cell 2021;184(5):1127-1132. DOI: 10.1016/j.cell.2021.01.044.

4. Ramanathan M, Ferguson ID, Miao W, Khavari PA. SARS-CoV-2 B.1.1.7 and B.1.351 spike variants bind human ACE2 with increased affinity. Lancet Infect Dis 2021;21(8):1070. DOI: 10.1016/S1473-3 099(21)00262-0.

5. Contou D, Fraisse M, Pajot O, Tirolien JA, Mentec H, Plantefeve G. Comparison between first and second wave among critically ill COVID-19 patients admitted to a French ICU: no prognostic improvement during the second wave ? Crit Care 2021;25(1):3. DOI: 10.1186/s13054-020-03449-6.

6. Karagiannidis C, Windisch W, McAuley D, Welte T, Busse R. Major differences in ICU admissions during first and second COVID-19 wave in Germany. Lancet Resp Med 2021;9(5):E47-E48. DOI: 10.1016/S2213-26 00(21)00101-6.

7. Herold T, Jurinovic V, Amreich C, Lipworth BJ, Hellmuth JC, von Bergwelt-Baildon $M$, et al. Elevated levels of IL- 6 and CRP predict the need for mechanical ventilation in COVID-19. J Allergy Clin Immunol 2020;146(1):128-136.e4. DOI: 10.1016/j.jaci.2020.05.008.

8. Kartz LM. (A little) Clarity on convalescent plasma for COVID-19. N Eng J Med 2021;384:666-668. DOI: 10.1056/NEJMe2035678.

9. Government of India Ministry of Health and Family Welfare Director General of Health Services (EMR Division). Clinical management protocol: COVID-19; version 3.0. 2020. Available from: https://mohfw. gov.in.

10. Government of India Ministry of Health and Family Welfare. Clinical management protocol: COVID-19; version 6.0. 2021. Available from: https://www.mohfw.gov.in/pdf/ COVID19ClinicalManagementProtocolAlgorithmAdults19thMay2021. pdf.

11. Chakraborty C, Sharma AR, Bhattacharya M, Agoramoorthy G, Lee SS The current second wave and COVID-19 vaccination status in India. Brain Behav Immun 2021;96:1-4. DOI: 10.1016/j.bbi.2021.05.018.

12. Fisman DN, Tuite AR. Progressive increase in virulence of novel SARS CoV-2 variants in Ontario, Canada. medRxiv 2021:2021.07.05.21260050. DOI: 10.1101/2021.07.05.21260050.

13. Galasso V, Pons V, Profeta P, Becher M, Brouard S, Foucault M. Gender differences in COVID-19 attitudes and behavior: panel evidence from eight countries. Proc Natl Acad Sci USA 2020;117(44):27285-27291. DOI: 10.1073/pnas.2012520117.

14. Sanyaolu A, Okorie C, Marinkovic A, Patidar R, Yonis K, Desai P, et al. Comorbidity and its impact on patients with COVID-19.SN Compr Clin Med 2020;2:1069-1076.

15. Guan $W-J$, Liang $W-H$, Zhao $Y$, Liang $H$, Chen $Z$, Li Y, et al. Comorbidity and its impact on 1590 patients with COVID-19 in China: a nationwide analysis. Eur Respir J 2020;55(5):2000547. DOI: 10.1183/13993003.00547-2020.

16. Liang X, Shi Li, Wang Y, Xiao W, Duan G, Yang H, et al. The association of hypertension with severity and mortality COVID-19 patients: evidence based on adjusted effect estimates. J Infect 2020;81(3):e44-e47. DOI: 10.1016/j.jinf.2020.06.060.

17. ZOE COVID Study. Available from: https://covid.joinzoe.com/post/ new-top-5-covid-symptoms.

18. Sudre $\mathrm{CH}$, Keshet $A$, Graham MS, Joshi AD, Shilo S, Rossman $\mathrm{H}$, et al. Anosmia, ageusia, and other COVID-19 like symptoms in association with a positive SARS-CoV-2 test across six national digital surveillance platforms:anobservational study.LancetDigitHealth 2021;3(9):e577-e5 86. DOI: $10.1016 /$ S2589-7500(21)00115-1.

19. Von Bartheld CS, Hagen MM, Butowt R. The D614G virus mutation enhances anosmia in COVID-19 patients: evidence from asystematic review and meta-analysis of studies from south Asia. ACS Chem Neurosci 2021;12(9):3535-3549. DOI: 10.1021/ acschemneuro.1c00542.

20. RECOVERY Collaborative Group; Horby P, Lim WS, Emberson JR, Mafham M, Bell JL, Linsell L, et al. Dexamethasone in hospitalized 
patients with COVID-19-prelimnary report. N Engl J Med 2021;384(8):693-704. DOI: 10.1056/NEJMoa2021436.

21. Ko JJ, Wu C, Mehta N, Wald-Dickler N, Yang W, Qiao R. A comparison of methylprednisolone and dexamethasone in intensive care patients with COVID-19. J Intensive Care Med 2021;36(6):673-680. DOI: $10.1177 / 0885066621994057$.

22. Ranjbar K, Moghadami M, Mirahmadizadeh A. Methylprednisolone or dexamethasone, which one is superior corticosteroid in the treatment of hospitalized COVID-19 patients: a triple-blinded randomized controlled trial. BMC Infect Dis 2021;21(1):337. DOI: 10.1186/s12879-021-06045-3.

23. Wang Y, Lu X, Li Y, Chen H, Chen T, Su N, et al. Clinical course and outcomes of 344 intensive care patients with COVID-19. Am J Respir Crit Care Med 2020;201(11):1430-1434. DOI: 10.1164/rccm.202003-07 36LE.

24. Lim ZJ, Subramaniam A, Reddy MP, Blecher G, Kadam U, Afroz A, et al. Case fatality rates for patients with COVID-19 requiring invasive mechanical ventilation. A meta-analysis. Am J Respir Crit Care Med 2021;203(1):54-66. DOI: 10.1164/rccm.202006-2405OC.

25. Tobin MJ, Jubran A, Laghi F. Non-invasive strategies in COVID-19: epistemology, randomised trials, guidelines, physiology. Eur Respir J 2021;57(2):2004247. DOI: 10.1183/ 13993003.04247-2020.

26. Doidge JC, Gould DW, Ferrando-Vivas P, Mouncey PR, Thomas K, Shankar-Hari $M$, et al. Trends in intensive care for patients with COVID-19 in England, Wales, and Northern Ireland. Am J Respir Crit Care Med 2021;203(5):565-574. DOI: 10.1164/rccm.202008-3212OC.

27. Wang Z, Wang Y, Yang Z, Wu H, Liang J, Liang H, et al. The use of non-invasive ventilation in COVID-19: a systematic review. Int J Infect Dis 2021;106:254-261. DOI: 10.1016/j.jijid.2021.03.078.
28. Li J, Fink JB, Ehrmann S. High-flow nasal cannula for COVID-19 patients: low risk of bio-aerosol dispersion. Eur Respir J 2020;55(5):2000892. DOI: 10.1183/1399003.00892-2020.

29. Mukhtar A, Lotfy A, Hasanin A, El-Hefnawy I, ElAdawy A. Outcome of non-invasive ventilation in COVID-19 critically ill patients: a retrospective observational study. Anaesth Crit Care Pain Med 2020;39(5):579-580. DOI: 10.1016/j.accpm.2020.07.012.

30. Bellani G, Grasselli G, Cecconi M, AntoliniL, Borelli M, De Giacomi F, et al. Noninvasive ventilatory support of patients with COVID-19 outside the intensive care units (ward-COVID). Ann Am Thorac Soc 2021;18(6):1020-1026. DOI: 10.1513/AnnalsATS.202008-10800C.

31. Bravata DM, Perkins AJ, Meyers LJ, Arling G, Zhang Y, Zillich AJ, et al. Association of intensive care unit patient load and demand with mortality rates in US Department of Veterans Affair hospitals during the COVID-19 pandemic. JAMA Newt Open 2021;4(1):e2034266. DOI: 10.1001/jamanetworkopen2020.34266.

32. Taccone FS, Goethem NV, Pauw R, Wittebole X, Blot K, Van Oyen H, et al. The role of organizational characteristics on the outcome of COVID-19 patients admitted to the ICU in Belgium. Lancet Regional Health-Europe 2020. DOI: 10.1016/j.lanepe.2020.100019.

33. Zhou F, Yu T, Du R, Fan G, Liu Y, Liu Z, et al. Clinical course and risk factors for mortality of adult inpatients with COVID-19 in Wuhan, China: a retrospective cohort study. Lancet 2020;395:1054-1062. DOI: 10.1016/S0140-6736(20)30566-3.

34. Thomson RJ, Hunter J, Dutton J, Schneider J, Khosravi M, Casement A, et al. Clinical characteristics and outcomes of critically ill patients with COVID-19 admitted to an intensive care unit in London: a prospective observational cohort study. PLoS One 2020;15(12):e0243710. DOI: 10.1371/journal.pone.0243710. 